\title{
An efficient analytical method for dimensioning of CDMA cellular networks serving streaming calls
}

\author{
Bartłomiej Błaszczyszyn \\ INRIA \& Ecole Normale Supérieure and \\ Mathematical Institute University of Wrocław \\ 45 rue d'Ulm \\ 75005 Paris France \\ Bartek.Blaszczyszyn@ens.fr
}

\author{
Mohamed Kadhem Karray \\ France Telecom, Research and Development \\ Division \\ $38 / 40$ rue du Général Leclerc \\ 92794 Issy-les-Moulineaux France \\ mohamed.karray@orange-ftgroup.com
}

\begin{abstract}
We propose an efficient analytical method for dimensioning of the downlink radio part of CDMA networks serving real-time calls. The proposed method is based on some admission condition called average feasibility condition (AFC). The advantage of using this condition is twofold: It has the well-known multi-Erlang form making the corresponding call blocking probabilities easy to evaluate, using e.g. KaufmanRoberts algorithm. Moreover, it approximates the necessary and sufficient condition of the feasibility of power allocation (NSFC) that gives intrinsic, ultimate limitation of the network performance. More precisely, our AFC is some modification of the distributed sufficient condition of the power allocation proposed in [1] which, in its original form, is too conservative yielding a loss of capacity compared to NSFC of about $25 \%$ (for the voice traffic). The modification consists of replacing the other-base-station maximal power limitation by an average emitted power approximation evaluated in some simple yet pertinent mean network model. We analytically evaluate the call blocking probabilities in the network model with Poisson arrival stream controlled by AFC. Moreover, in order to validate the pertinence of the proposed approach in cellular network dimensioning process, we compare the obtained blocking probabilities to those estimated from simulations of the model running NSFC (in the regimes of interest for the dimensioning process). This comparison shows that AFC yields a gap of capacity of about $5 \%$ (for the voice traffic).
\end{abstract}

\section{Categories and Subject Descriptors}

D.2.8 [Software Engineering]: Metrics-performance measures

\section{General Terms}

Performance

\footnotetext{
Permission to make digital or hard copies of all or part of this work for personal or classroom use is granted without fee provided that copies are not made or distributed for profit or commercial advantage and that copies bear this notice and the full citation on the first page. To copy otherwise, to republish, to post on servers or to redistribute to lists, requires prior specific permission and/or a fee.

ValueTools 2008 October 21-23, 2008, Athens, GREECE.

Copyright (C) 2008 ICST ISBN \# 978-963-9799-31-8.
}

\section{INTRODUCTION}

Operators of cellular networks aim to minimize the number of deployed base stations (or, equivalently, to maximize their coverage regions) given the required quality of service of their users. This is called the dimensioning problem. The inputs of this problem are essentially the traffic demand (in Erlang per surface unit) and the required quality of service. In the case of a cellular network serving real-time services (such as voice calls, video streaming, etc.) this quality of service may be identified with the blocking probability which should not exceed typically $2 \%$. If one knows the relation between the blocking probability, the base station coverage radius (called cell radius) and the traffic demand, then the dimensioning problem may be solved in principle. For an efficient dimensioning method, this relation should obviously be accurate enough and relatively easy to evaluate (rapid) in order to facilitate the optimization problem. The former requirement is generally satisfied by existing methods based on the network simulation. However solving a complex optimization problem by means of long simulations is not efficient. On the other hand the known explicit analytical methods for the network evaluation are not yet sufficiently accurate. In this paper we propose some break-through in this matter considering the downlink of a CDMA cellular network serving real-time services.

More precisely, we propose an efficient analytical dimensioning method based on some "virtual" admission condition called average feasibility condition (AFC). The blocking probabilities induced by this condition are close to those observed for existing networks, implementing some "real" but complex and difficult to evaluate control schemes proposed by various manufacturers. This will be explained in what follows.

Network control versus network dimensioning. In a cellular network, each base station transmits some power to each user in its cell. In doing so, two constraints should be respected. Firstly, we have a power limit constraint saying that the power transmitted by each base station should not exceed some given threshold. Secondly, we have a Shannon type constraint saying that the Signal-to-Interference-andNoise-Ratio (SINR) of each user should exceed some threshold. This latter depends on the user bit-rate, the channel characteristics (such as fading) and the system bandwidth. The problem of finding a vector of powers (to be dedicated to the individual users), which respects the above two constraints is called power allocation problem. If this problem is feasible, i.e. admits a solution, then all the users in the network may be served at their required bit-rates. Other- 
wise, at least one call in the network should be interrupted. We say in this case that the power allocation problem is unfeasible.

In order to cope with feasibility of the power allocation problem, some complex control schemes are implemented in real networks. Roughly speaking, they consist of checking at the arrival instant of each new user some admission condition allowing to decide whether it can be admitted or not. An "ideal" admission control scheme should admit a new user if and only if the power allocation problem with this new user is feasible. Implementing such a control scheme based on necessary and sufficient feasibility condition (NSFC) would be optimal since leading to a minimal blocking probability (among the family of control schemes assuring power allocation feasibility). Unfortunately NSFC may not be implemented in a real network since it is not distributed; i.e., it requires the information about the radio conditions of all the users in the network

Several practical schemes are implemented by network controller manufacturers (see e.g. [12], [15]). These schemes are decentralized; i.e., each base station may take the admission decision locally without requiring information about the current state of other base stations (such as the number, positions, radio conditions of their users). They only approximately assure the feasibility of the power allocation problem, however these approximations are accurate enough, in particular for small bit-rates (such as those required for voice), and for small cells (deployed typically in urban areas); cf. [13, p.231] for a comparison of these schemes with NSFC

As we already said, in order to solve the network dimensioning problem one needs an accurate and rapid method for the evaluation of the blocking probabilities induced by the admission control schemes implemented in the network controllers. Unfortunately, these blocking probabilities do not admit any analytical analysis and need to be estimated using time consuming simulations, which are not suitable for network dimensioning. The same holds true for the performance of the "ideal" network model implementing the NSFC condition.

Existing dimensioning methods are generally based on some network models using some alternative, simplified, "virtual" admission conditions. In order for the dimensioning method to be rapid, these conditions are chosen to have the so-called multi-Erlang form: the sum of some function of user radio conditions (principally determined by its geographic location) and its required bit rate evaluated over all users in each cell should not exceed some constant. This makes the corresponding call blocking probability easy to evaluate, using e.g. Kaufman-Roberts algorithm. The main concern of this approach is about the relation between the performance of the "real" and the "virtual" admission conditions. In order to make them related to each other, the virtual admission conditions should be close to the NSFC, as it is the case for the real admission conditions.

Our dimensioning method. The AFC proposed in the present paper is some modification of the sufficient feasibility condition (SFC) for the power allocation proposed in $[1$ which has the multi-Erlang form. Unfortunately, SFC is too conservative yielding about $20 \%$ of capacity loss (see [13, §3.1]). The modified AFC consists of replacing the otherbase-station maximal power limitation used in SFC by an approximation of the average emitted power evaluated in some simple yet pertinent mean network model that essen- tially consists of averaging over the geometry of the Poisson distribution of users.

We analytically evaluate the call blocking probabilities in the network model with Poisson arrival stream controlled by our AFC. Moreover, in order to validate the pertinence of the model in cellular network dimensioning process, we compare the obtained blocking probabilities in the region of interest for the dimensioning to those estimated from simulations of the model running NSFC.

We develop our approach for the downlink of a CDMA network serving streaming calls that are not moving during the service. However this approach can be extended in principle to take into account the uplink, user mobility, as well as the elastic bit rate. Indeed, the sufficient feasibility condition has already been studied in these cases (see $[6,5$, 13])

Paper organization. The remaining part of this paper is organized as follows. In Section 2 we briefly review the related work and position our contribution in this context. Section 3 recalls the Erlang formula in a general context of evaluation of the blocking probabilities in cellular networks. In Section 4 we describe some specific aspect of power controlled networks (as CDMA): power allocation problem, a necessary and sufficient condition for its feasibility, and a mean model. Section 5 first brings a review of already proposed admission conditions and then introduces AFC that is the main idea of the present paper. The numerical results comparing the performance of different admission conditions are presented in Section 6. More detailed description of the network model (requiring further notation), as well as explicit expressions of the quantities building the considered admission conditions are deferred to the Appendix.

\section{RELATED WORKS}

Since we are interested in dimensioning of the radio part of the network, we shall only review literature directly related to this problem. In particular, we shall not comment here on practically implemented control schemes.

Several dimensioning methods for CDMA networks have been proposed in the literature. The authors of $[10,19,16$, $18,9]$ propose dimensioning methods based on the so-called outage probability. This notion corresponds to the probability that the SINR of a given user doesn't exceed some threshold, when customers are modelled by a static Poisson configuration of points. Some other dimensioning methods are based on the so-called pole capacity (cf [19]). The drawback of these methods is that neither the outage probability nor the pole capacity are related in a straight-forward way to the blocking probability that should account for a dynamic process of users arriving and being either blocked or admitted for the service.

Publications [19] and [7] propose methods to calculate the blocking probability, but they concern, respectively, the uplink and the case of elastic bit-rate calls and thus are not directly relevant to the present study.

The fundamental papers $[21,20]$ show how the power allocation problem without power limitations can be reduced to an algebraic system of linear inequalities. Moreover, they recognize that the spectral radius of the (non-negative) matrix corresponding to this system not greater than 1 is the necessary and sufficient condition of the feasibility of power allocation without power limitations.

The approach of $[21,11]$ is continued in $[3,13]$ where a sufficient feasibility condition (SFC) for the feasibility of the 
power allocation problem is proposed. The blocking probability induced by this SFC is an upper bound of this induced by NSFC ${ }^{1}$. Unfortunately, as we will show in the numerical section, the SFC blocking is not always close to NSFC, particularly for a range of blocking probabilities which is precisely relevant for dimensioning. Nevertheless, since SFC gives an upper bound of the blocking probability, it leads to a conservative dimensioning.

An analytic method proposed in [8], that we call the "constantload method" (see $\S 5.3$ for more details) is rapid, but not sufficiently accurate as we shall see in the numerical section. The main concern about it is that one does not know a priori how to fix its crucial parameter, called load parameter. A modification of this method proposed in [4] consists of some 'iterations' of the 'constant-load method' aiming to approximate iteratively the load parameter and thus the blocking probability. However, it is not clear how to calculate the load from the expressions presented in [4]. Moreover the convergence of these iterations to NSFC is not shown neither analytically nor numerically.

Position of our contribution in this context. Our approach via the AFC condition is strongly related to the SFC condition presented in [3, 1]; (see also [13]) whose blocking probability was evaluated via a spatial version of the Erlang's loss formula in [2]. It can also be seen as a modification of the constant load parameter approach [8, 4], in which the load parameter is neither fixed arbitrarily nor approximated iteratively, but evaluated by means of the mean network model proposed and evaluated in [3]. This allows to capture all important network and traffic parameters in a simple manner.

\section{BLOCKING PROBABILITY}

In order to evaluate the blocking probability it is necessary to specify the dynamics of call arrivals and durations as well as identify the set of feasible configuration of users (allowed by a given admission condition).

\subsection{Traffic demand}

We model a cellular network as a bounded subset $\mathbb{D}$ of the plane $\mathbb{R}^{2}$ partitioned into cells. The inter-arrival times of calls are assumed to be independent and identically distributed (i.i.d.) exponential random variables with rate $\lambda$ (mean $\frac{1}{\lambda}$ ). The position of each arriving user is picked at random in $\mathbb{D}$ according to some distribution $Q(d x)$ (which we take uniform for the numerical evaluations). We assume that users don't move during their calls. Each call requires to be served by the network at a given bit-rate during some service time. The durations of the different calls are assumed to be i.i.d. exponentially distributed with mean $\frac{1}{\mu}$. (This assumption may be relaxed due to the so-called insensitivity property, but this is not in the scope of the present paper.) The quantity $\rho(\mathbb{D})=\frac{\lambda}{\mu}$ is called the traffic demand (expressed in Erlangs) in the whole network.

The set of positions of all users served at a given time is called configuration of users. In accordance with the point process formalism it is customary to identify configurations of users with finite counting measures on $\mathbb{D}$, assuming that each user corresponds to a unit (Dirac) measure concentrated at its location. Let $\mathbb{M}$ be the set of such measures.

\footnotetext{
${ }^{1}$ We are not aware of any published proof of this very intuitive statement confirmed by simulations.
}

We denote by $\left\{N_{t}\right\}_{t>0}$ the process describing the evolution in time of the user configurations in $\mathbb{D}$ (due to arrivals and departures) in the absence of any admission control. We call it free process. Due to our convention it takes its values in $\mathbb{M}$.

By our previous assumptions the free process $\left\{N_{t}\right\}_{t>0}$ is a Markov process which is ergodic with stationary invariant distribution $\Pi$ corresponding to the Poisson point process on $\mathbb{D}$ with mean measure $\rho(\mathbb{D}) Q(d x)$. In other words: the stationary free process (offered traffic) of positions of users is Poisson with mean measure equal to the traffic demand. Moreover $\left\{N_{t}\right\}_{t>0}$ is reversible with respect to $\Pi$.

\subsection{Erlang's loss formula}

We assume that a given admission condition consists of verifying whether a given configuration of users with a new arrival belongs to some set of feasible configurations $\mathbb{M}^{\mathrm{f}}$. We tacitly assume also that no user departure from a feasible configuration can make it unfeasible.

Denote the evolution of the free process modified (controlled) by the given admission condition by $\left\{N_{t}^{\mathrm{tb}}\right\}_{t \geq 0}$. Due to the form of the admission condition this process is also Markov. More precisely, it has the same dynamics as the free process except that the transitions (i.e. arrivals) that would lead outside $\mathbb{M}^{\mathrm{f}}$ are blocked ${ }^{2}$. Such a modification of the Markov process is called truncation to $\mathbb{M}^{f}$. The crucial observation, made by the reversibility of the free process, is that the truncated process $\left\{N_{t}^{\text {tb }}\right\}_{t>0}$ admits as its invariant distribution the truncation of $\Pi$ to $\mathbb{M}^{\mathrm{f}}$; that is, $\Pi^{\mathrm{tb}}(\Gamma)=\Pi\left(\Gamma \cap \mathbb{M}^{\mathrm{f}}\right) / \Pi\left(\mathbb{M}^{\mathrm{f}}\right)$ for $\Gamma \subset \mathbb{M}$.

The blocking probability (in some region of the network) is defined as the proportion of the blocked calls to the total number of arrivals arriving to this region in the long run of the system. The celebrated Erlang's loss formula allows to express this ergodic average by means of the invariant measure of the free process and in our spatial point process formalism takes the form $b_{x}=\Pi\left(\left\{\nu \in \mathbb{M}^{\mathrm{f}}: \nu+\epsilon_{x} \notin\right.\right.$ $\left.\left.\mathbb{M}^{\mathrm{f}}\right\}\right) / \Pi\left(\mathbb{M}^{\mathrm{f}}\right)$, where $b_{x}$ is the blocking rate of users arriving at the location $x \in \mathbb{D}(\mathrm{cf}[2])$. Note that, in analogy to the classical version of the Erlang's loss formula, the blocking rate $b_{x}$ of users arriving at $x$ is equal to the conditional probability that the stationary configuration of users in the free Poisson process cannot admit a new user at $x$ given this configuration is in $\mathbb{M}^{\mathrm{f}}$. Integrating $b_{x}$ against the distribution $Q(d x)$ over some given subset of the network $\mathbb{D}$ gives the blocking probability in this set (region) of the network (cf $[2])$.

For this formula to be of any use in the dimensioning process one needs an efficient way of evaluating the Poisson probabilities in the numerator and the denominator, in particular $\Pi\left(\mathbb{M}^{\mathrm{f}}\right)$. Such efficient method exists for some particular form of the admission condition as we explain in what follows.

Let $\mathbf{U}$ be the set of base stations which we assume finite. In order to simplify notation we use the same letter, for example $u \in \mathbf{U}$, to designate the base station and its cell, i.e. the subset of $\mathbb{D}$ served by the base station. We say that the admission condition has the multi-Erlang form if the corresponding set of feasible configurations $\mathbb{M}^{\mathrm{f}}$ has the following form

$$
\mathbb{M}^{\mathrm{f}}=\bigcap_{u \in \mathbf{U}}\left\{\nu \in \mathbb{M}: \int_{u} \varphi(x) \nu(d x)<C_{u}\right\}
$$

\footnotetext{
${ }^{2}$ The superscript 'tb' in $N_{t}^{\mathrm{tb}}$ stands for 'transition blocking'.
} 
where the integral should be interpreted as the sum of the values of some function $\varphi$ of user location $x$ (and possibly its other characteristics as e.g. bit-rate) evaluated over all users in cell $u$ and $C_{u}$ is some constant, possibly dependent on the base station. In this case, we may easily evaluate the blocking probability by discretization of $\mathbb{D}$ and using the Kaufman-Roberts algorithm [14, 17].

\subsection{Applications of the blocking probability for- mula}

We have already said that an accurate expression of the blocking probability as a function of the traffic demand and the cell radius permits to solve the dimensioning problem. It permits also to solve some other related interesting problems as well. Assume that we have an expression $b=b(\rho, R)$ of the blocking probability $b$ as a function of the traffic demand per surface unit $\rho$ and the cell radius $R$. Such relation permits to solve the following problems:

- Consider an existing network with given cell radius $R$ and traffic demand $\rho$ per surface unit. The quality of service perceived by the users may be evaluated by the blocking probability $b=b(\rho, R)$.

- Consider an existing network with given cell radius $R$. The capacity of the network at the blocking probability $b$ may be defined as the traffic demand $\rho$ corresponding the solution of the equation $b(\rho, R)=b$ in $\rho$.

- Assume that an operator has to construct a network satisfying a given traffic demand $\rho$ with a blocking probability at most $b$. He has to calculate the cell radius such that $b(\rho, R)=b$. This is the dimensioning problem.

- Since the network cost is proportional to the number of base stations per unit of surface $1 /\left(\pi R^{2}\right)$, then minimizing the cost is equivalent to dimensioning.

\section{POWER ALLOCATION PROBLEM}

In this section we describe some ingredients which shall be useful in the description of the admission conditions in the next section. We first formulate the power allocation problem, then we give a necessary and sufficient condition of its feasibility, and finally we describe the mean model.

\subsection{Problem formulation}

The allocation problem may be formulated as follows:

Does there exist a vector $\mathbf{P}=\left(P_{u}\right)_{u \in \mathbf{U}}$ of the powers transmitted by the base stations such that

$$
\left\{\begin{array}{l}
(\mathbf{1}-A) \mathbf{P} \geq a \\
0 \leq \mathbf{P} \leq \tilde{\mathbf{P}}
\end{array}\right.
$$

where $A=\left[A_{u v}\right]_{u, v \in \mathbf{U}}$ is a given non-negative matrix, $\mathbf{1}$ is the unitary matrix, $a=\left(a_{u}\right)_{u \in \mathbf{U}}$ is a given non-negative vector and $\tilde{\mathbf{P}}=\left(\tilde{P}_{u}\right)_{u \in \mathbf{U}}$ is the vector of maximal powers of base stations (cf. for example [13, Eq. (3.14) p.22]). (All the vectors are considered as column vectors and the inequality between two vectors is understood component-wise.) The explicit expressions of the entries $A$ and $a$ are given in Appendix $\S$ C.1.

\subsection{NSFC}

The following proposition gives a necessary and sufficient condition (NSFC) of the feasibility (i.e. existence of a solution) of the power allocation problem (see [13, Prop. 5 p.22]).
Proposition 1 (NSFC). Problem (2) is feasible (i.e. admits a solution) iff

$$
\left\{\begin{array}{l}
\boldsymbol{\rho}(A)<1 \\
(\mathbf{1}-A)^{-1} a \leq \tilde{\mathbf{P}}
\end{array}\right.
$$

where $\boldsymbol{\rho}(\cdot)$ designates the spectral radius. In this case, the minimal solution is

$$
\mathbf{P}=(\mathbf{1}-A)^{-1} a
$$

Note that building an admission control scheme based on NSFC would require calculation of the spectral radius of the matrix $A$ that depends on the positions of the users in all the network. Such centralized scheme is not practical in real networks. Neither NSFC has the multi-Erlang form (1).

\subsection{Mean model}

Recall that the admission condition NSFC implies that the transmitted power is given by (4). The average transmitted power may be expressed as follows

$$
\bar{P}_{\mathrm{R}}=E_{\Pi^{\mathrm{tb}}}\left[(\mathbf{1}-A)^{-1} a\right]
$$

where $\Pi^{\mathrm{tb}}$ is the stationary distribution of users in the "ideal" network model implementing NSFC and $E_{\Pi^{\mathrm{tb}}}[\cdot]$ represents the expectation with respect to this distribution. This expectation is difficult to calculate. Our idea is to approximate it by

$$
\bar{P}_{0}:=\left(\mathbf{1}-E_{\Pi}[A]\right)^{-1} E_{\Pi}[a]
$$

where the expectation is taken with respect to the free process $\Pi$ (which is in fact a Poisson process on $\mathbb{D}$ ). The righthand side of the above equation is well defined only when the spectral radius $\boldsymbol{\rho}\left(E_{\Pi}[A]\right)<1$. Otherwise we let by convention $\bar{P}_{0}:=\infty$

Note that the right-hand sides of the two above equations differ in two points. Not only, the stationary distribution of the constrained process $\Pi^{\mathrm{tb}}$ is replaced by that of the free process $\Pi$, but also the expectation is taken at different levels.

In order to account for the fact that the average transmitted power should not exceed the maximal transmission power, we propose the following further approximation of $\bar{P}_{\mathrm{R}}$

$$
\bar{P}:=\max \left(\bar{P}_{0}, \tilde{P}\right)=\max \left(\left(\mathbf{1}-E_{\Pi}[A]\right)^{-1} E_{\Pi}[a], \tilde{P}\right)
$$

We show in the appendix ( $\S$ C.2) that a detailed expression of $\bar{P}_{0}$ may be obtained in some particular cases which are relevant for network dimensioning.

\section{ADMISSION CONDITIONS}

We review now some already proposed admission conditions suitable for the network dimensioning and introduce AFC which is the main idea of the present paper.

\subsection{Manufacturers' schemes}

The manufacturers' admission control schemes are typically based on the powers transmitted by the base stations just before the arrival of a new user $[12,15]$. A new user arriving in a cell is admitted if the power of the corresponding base station is less than the maximal power multiplied by some given constant $k \in(0,1)$. Since the transmitted power may be approximated in a distributed way as shown in [20], this admission scheme is decentralized. Nevertheless it doesn't assure the feasibility of the power allocation problem. The comparison of the performance of this scheme to NSFC is presented in $[13$, p.231]. It is shown there that 
the manufacturers' scheme assures approximately the feasibility of the power allocation problem particularly for small bit-rates (such as voice), and for small cells (i.e. in urban areas). Moreover, in this context, its performance is close to NSFC. Hence it is well adapted for implementation in real networks. However its performance may not by easily evaluated. Hence it may not be used as an admission condition in the network dimensioning process.

In what follows we shall not present blocking probabilities related to the manufacturers' schemes tacitly assuming that they are close to these offered by NSFC.

\subsection{SFC}

The following proposition gives a sufficient feasibility condition (SFC) having the multi-Erlang form. This condition was proposed in $[1,13]$.

$$
\begin{array}{ll}
\text { Proposition 2 } & \text { (SFC). If } \\
& (\mathbf{1}-A) \tilde{\mathbf{P}} \geq a
\end{array}
$$

then (2) is feasible. $\mathbf{U}$,

Note that SFC (7) may be written as follows: for all $u \in$

$$
\left(1-A_{u u}\right) \tilde{P}_{u}-\sum_{v \neq u} A_{u v} \tilde{P}_{v} \geq a_{u}
$$

It is not obvious from the above formula that SFC has the multi-Erlang form. Indeed, this is due to the fact that for given base stations $u$ and $v$, the term $A_{u v}$ of the matrix $A$ (see expressions in $\S$ C.1) is a sum over the users served by base station $u$ of some function of their path losses with respect to base stations $u$ and $v$. Similar observation can be made for components $a_{u}$ of the vector $a$.

\subsection{Average feasibility condition (AFC)}

Since SFC implies NSFC, for a given traffic demand the blocking probability of SFC is larger than that of NSFC. As the traffic demand increases from 0 to infinity, these two blocking probabilities increase from 0 to 1 with a good fitting in extreme regions. Nevertheless there is an intermediate region where the difference between these two blocking probabilities is significant. Unfortunately this is precisely the interesting region when one dimensions the network.

In order to get more insight about what happens, observe that the Shannon constraint in the power allocation problem (first equation in (2)) may be written as follows, for each base station $u$,

$$
\left(1-A_{u u}\right) P_{u}-\sum_{v \neq u} A_{u v} P_{v} \geq a_{u}
$$

where we may see the term $\sum_{v \neq u} A_{u v} P_{v}$ as the interference contribution. Recall also that SFC given by (8) consists of replacing actual emitted powers $P_{u}$ and $P_{v}$ in (9) by their respective maximal values $\tilde{P}_{u}$ and $\tilde{P}_{v}$.

Comparing these two expressions for a very small traffic demand, as long as the blocking probability of SFC is null, that of NSFC is also null and hence both coincide in this region. On the other hand, for high traffic demand, when the powers emitted under NSFC $P_{u}$ and $P_{v}$ approach their maximal values, SFC is a good approximation of NSFC. In the intermediate case, the gap observed by simulations between SFC and NSFC is possibly caused by the overestimation of the interference contribution in SFC.

To overcome this drawback, using a heuristic reasoning, one can propose a modification of SFC which consists of replacing $P_{v}$ in the interference part of (9) by some arbitrary value $\bar{P}$ that is independent of $v$ (assume that the network is symmetric) and deterministic (not random). Thus we get

$$
\left(1-A_{u u}\right) P_{u}-\sum_{v \neq u} A_{u v} \bar{P} \geq a_{u}
$$

Evaluating the minimal power $P_{u}$ that should be emitted by the base station $u$ observing this new condition we get

$$
P_{u}=\frac{a_{u}+\sum_{v \neq u} A_{u v} \bar{P}}{1-A_{u u}}
$$

And applying the power limitation conditions $P_{u} \leq \tilde{P}_{u}$, one gets a new admission condition for base station $u$

$$
\left(1-A_{u u}\right) \tilde{P}_{u}-\sum_{v \neq u} A_{u v} \bar{P} \geq a_{u}
$$

The above condition may be viewed as a modification of the interference term $\sum_{v \neq u} A_{u v} \tilde{P}_{v}$ in SFC which consists of replacing $\tilde{P}_{v}$ by $\bar{P}$.

So far the value of $\bar{P}$ can be fixed arbitrarily. Indeed, taking it in some proportion to the maximal power

$$
\bar{P}=\ell \tilde{P}
$$

where $\ell$ is some fixed constant in $[0,1]$ (called load) we get the admission condition described in [8] called constant-load condition (CLC). It has the multi-Erlang form for the same reason as SFC. The drawback of this method is that it is not evident how to fix the load parameter $\ell$. In particular, simulations show that one fixed value of $\ell$ does not fit to different traffic demand/cell size scenarios.

To overcome this drawback a modification of CLC, proposed in [4], consists of some recursive evaluation of this parameter: for a given fixed value of $\ell$ the blocking probability corresponding to CLC is evaluated and supposed to be used to modify the value of $\ell$ in the next iteration. However, given this blocking probability, it is not clear how to calculate the transmitted powers in the expression for $\ell$ given in $[4] .^{3}$

By this time it should be evident that the value of $\bar{P}$ (equivalently $\ell$ ) has to depend on the network parameters and traffic demand in a simple yet pertinent manner. An interesting candidate for the parameter $\bar{P}$ (in the spirit of the law of large numbers) is the average power in the network applying the NSFC admission condition, which we denoted in $\S 4.3$ by $\bar{P}_{\mathrm{R}}$. We call the admission condition (10) with

$$
\bar{P}:=\bar{P}_{\mathrm{R}}
$$

the reference average feasibility condition (R-AFC). This condition is not practical, as it is written above, for dimensioning purpose, since it requires prior evaluation of $\bar{P}_{\mathrm{R}}$ by simulations. Nevertheless, we shall calculate its performance in the numerical section by simulations. We believe, it gives in some sense a bound on the performance we can hopefully obtain with an admission condition of the form (10) with $\bar{P}$ being mean emitter power.

\footnotetext{
${ }^{3}$ Indeed this expression depends on the positions of the users. In order to calculate the average of this expression over user positions, we need to know the distribution of these positions. If we assume a Poisson process with mean measure equal to the traffic demand, then the average power would be infinite. On the other hand calculation based on the truncation of the Poisson process to the set of configurations satisfying the CLC is not explicit and requires simulations. Moreover, the fact that the iterations proposed in [4] would converge to some average power and blocking probability approaching the NSFC ones is not demonstrated neither analytically nor numerically.
} 
Table 1: Capacity gap of the admission conditions $(R=0.525 \mathrm{~km})$.

\begin{tabular}{|l||c|c|c|c|}
\hline service $\backslash$ condition & CLC & SFC & R-AFC & AFC \\
\hline \hline voice $12.2 \mathrm{kbps}$ & -18 & 26 & -5 & 5 \\
\hline data $64 \mathrm{kbps}$ & 0 & 46 & -11 & -3 \\
\hline data $144 \mathrm{kbps}$ & 10 & 58 & -12 & -6 \\
\hline data $384 \mathrm{kbps}$ & 41 & 88 & -12 & -12 \\
\hline
\end{tabular}

Finally, in order to make the admission condition fully analytical, we propose to fix the value of $\bar{P}$ using the approximation $\bar{P}_{0}$ of $\bar{P}_{\mathrm{R}}$ obtained considering the mean model described in $\S 4.3$. This allows to capture all important network and traffic parameters in a simple manner. We shall validate in $\S 6$ that $\bar{P}_{0}$ is an acceptable approximation of $\bar{P}_{\mathrm{R}}$ (at least in the region of interest for dimensioning). We call the admission condition (10) with $\bar{P}$ is given by (6) the average feasibility condition (AFC). It has the multi-Erlang form, by the same argument as used for SFC. We will show in $\S 6$ that its performance approximates well that of NSFC in the region of interest for dimensioning of cellular networks.

\section{NUMERICAL RESULTS}

We have already observed that the dimensioning problem may be solved if we know the relation between the blocking probability, the traffic demand and the cell radius. Using the network model described in $\S \mathrm{B}$, we shall analyze this relation by representing the blocking probability as function of the traffic demand for different cell radii. We aim to compare the results of different admission conditions: NSFC, CLC, SFC, R-AFC and AFC. The results for NSFC and R-AFC are evaluated by simulations (simulating the traffic demand process described in $\S 3.1$ subject to the respective admission conditions), whereas those for CLC, SFC and AFC are obtained using Kaufman-Roberts algorithm (see $\S 3.2$ with the particular incarnation of the feasibility condition in (1) given by (C.3)).

We have already said that the blocking probability of interest for dimensioning is about $2 \%$. Nevertheless, we shall analyze the results in the range of blocking probability $[0,30 \%]$ with a particular attention around $2 \%$. To this end, we define the capacity as the traffic demand corresponding to the blocking probability $2 \%$. We define also the capacity gap as the relative difference between the capacity of a given admission condition, say $c$, and the capacity of NSFC, denote it $c_{\mathrm{R}}$; that is,

$$
\text { capacity gap: }=\frac{c-c_{\mathrm{R}}}{c_{\mathrm{R}}} \times 100 \quad(\text { expressed in \%) }
$$

We assume numerical values of engineering parameters corresponding to UMTS standard (see $\S$ B.5). In particular we consider four classes of services: voice calls at $12.2 \mathrm{kbps}\left({ }^{4}\right)$ and data streaming at $64,144,384 \mathrm{kbps}$. We evaluate the blocking probability for cell radii $R=0.525 \mathrm{~km} 3 \mathrm{~km}$ and $5 \mathrm{~km}$, but only the curves corresponding to $R=0.525 \mathrm{~km}$ are presented in the paper, because the other curves are similar. However the numerical values of the capacity gap are given for all three values of the cell radii.

\subsection{Discussion}

Figures 1, 2, 3, 4 represent the blocking probability as a function of the traffic demand for the above four streaming services respectively. In each figure, we assume that the

${ }^{4}$ The abbreviation kbps designates "Kilo-bit per second".

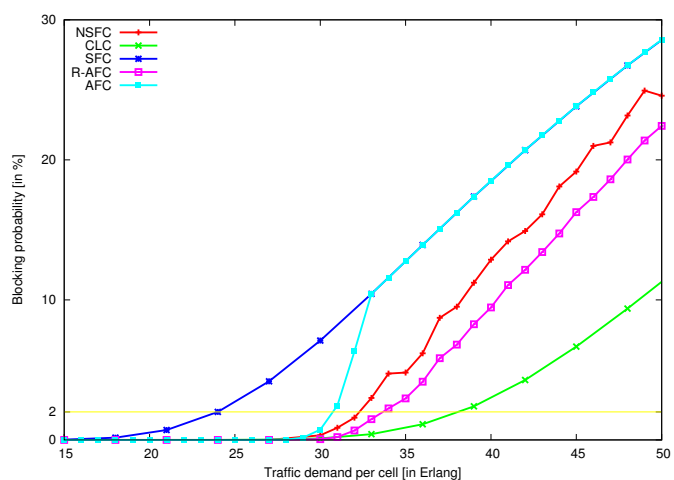

Figure 1: voice $12.2 \mathrm{kbps}$

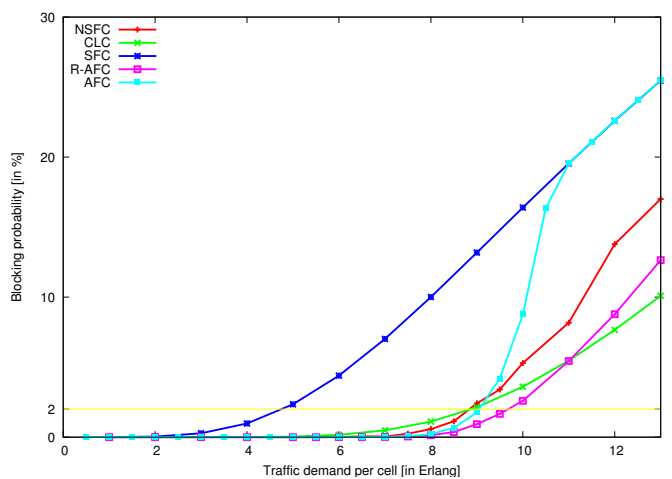

Figure 2: data $64 \mathrm{kbps}$

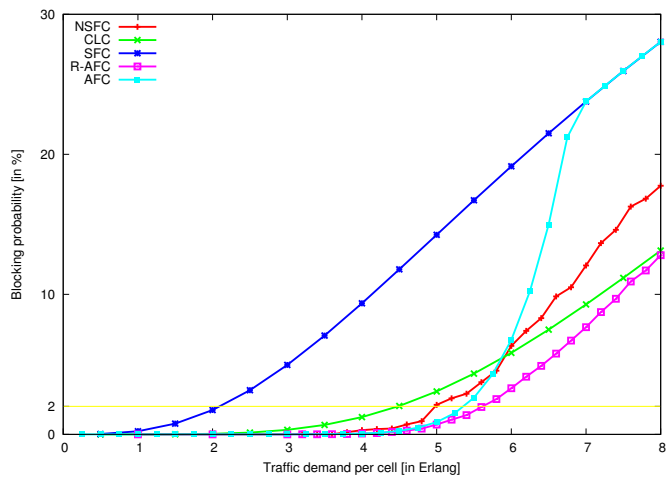

Figure 3: data $144 \mathrm{kbps}$

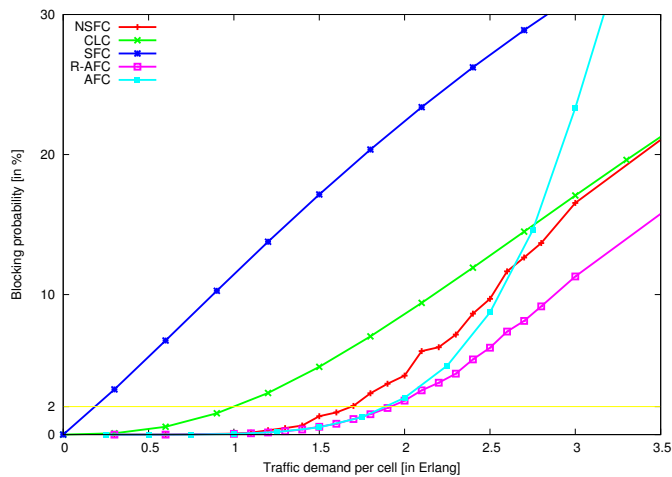

Figure 4: data $384 \mathrm{kbps}$

network serves users of a given traffic class only. Table 1 , derived from these figures, exhibits the capacitiy gaps of the different admission conditions. 
Table 2: Capacity gap of the admission conditions $(R=3 \mathbf{k m})$.

\begin{tabular}{|l||c|c|c|c|}
\hline service $\backslash$ condition & CLC & SFC & R-AFC & AFC \\
\hline \hline voice $12.2 \mathrm{kbps}$ & -16 & 25 & -3 & 3 \\
\hline data $64 \mathrm{kbps}$ & 0 & 43 & -8 & -3 \\
\hline data $144 \mathrm{kbps}$ & 13 & 56 & -10 & -6 \\
\hline data $384 \mathrm{kbps}$ & 43 & 88 & -8 & -8 \\
\hline
\end{tabular}

Table 3: Capacity gap of the admission conditions $(R=5 \mathrm{~km})$.

\begin{tabular}{|l||c|c|c|c|}
\hline service $\backslash$ condition & CLC & SFC & R-AFC & AFC \\
\hline \hline voice $12.2 \mathrm{kbps}$ & -12 & 18 & -3 & -3 \\
\hline data $64 \mathrm{kbps}$ & 3 & 38 & -10 & -10 \\
\hline data $144 \mathrm{kbps}$ & 10 & 50 & -13 & -17 \\
\hline data $384 \mathrm{kbps}$ & 48 & 76 & -38 & -38 \\
\hline
\end{tabular}

$S F C$. Observe first that the SFC, as expected, always gives the blocking probability larger than that induced by NSFC. This is a highly desirable property as it leads to conservative bounds on the network capacity and safe dimensioning decisions. However, the capacity loss due to the usage of SFC can go from $26 \%$ to $88 \%$, which is too much to use this condition in the dimensioning process.

CLC. The blocking probability induced by CLC depends very much on the value of the load parameter $\ell$. We fix its value at $\ell=0.5$ and observe that the corresponding blocking probability, depending on the traffic and bit-rate, overor under-estimate this induced by NSFC only loosely following it shape. The capacity gap of this condition varies from $-18 \%$ to $41 \%$. In order to suppress of the undesirable capacity over-estimation (negative values of the capacity gap) we take $\ell=0.75$ but then the capacity gap can go up to $71 \%$.

$R-A F C$. We study now the R-AFC condition. Recall that it corresponds to the situation when the load parameter is related to the mean emitted power estimated from the simulation of the network model implementing the NSFC. Thus it does not allow yet for a fully analytic dimensioning method. However, it is supposed to indicate the ultimate pertinence of the method based on the load parameter depending on the mean transmitted power. More precisely we believe that $\mathrm{R}$ AFC gives a bound on the performance one can hopefully obtain with an admission condition of the form (10), where $\bar{P}$ is an estimation of the mean transmitted power.

The first observation is that it is relatively close to the curve of the NSFC. Unfortunately it slightly underestimates it (one would prefer a conservative bound). However the absolute capacity gap is not too large and in particular much smaller than this of AFC and CLC (from $-5 \%$ to $-12 \%$ ). Also, the fact that the underestimation is systematic slightly ameliorates the situation allowing some universal correcting factor.

$A F C$. Recall that we see this condition as a further modification of the R-AFC, were we approximate the mean emitted power by using the mean network model (see $\S$ C.2) in order to make AFC analytically treatable. In order to validate this step we compare first in Figures 5, 6 the value of $\bar{P}$ used in AFC (i.e., given by the mean model) to $\bar{P}_{\mathrm{R}}$ estimated from

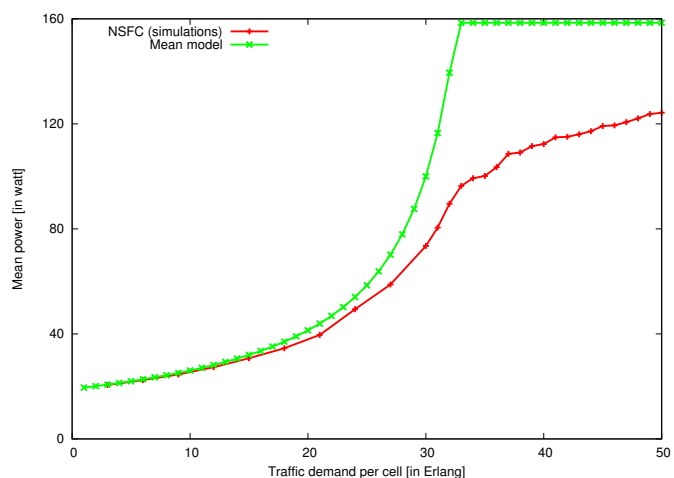

Figure 5: voice $12.2 \mathrm{kbps}$

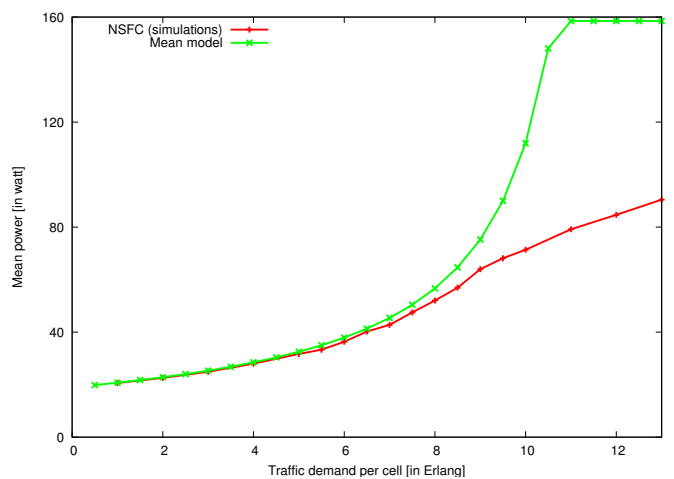

Figure 6: data $64 \mathrm{kbps}$

the simulation of the NSFC. These figures represent the average power as a function of the traffic demand for voice at $12.2 \mathrm{kbps}$ and data at $64 \mathrm{kbps}$. The results for data at 144 and $384 \mathrm{kbps}$ are analogous. We see that the quality of the proposed approximation is good when the traffic demand is small. As the traffic demand increases, the two curves diverge and the difference becomes significant when the mean power given by the mean model attains the maximal power. (Recall that from this point on the AFC condition coincides with the SFC). Fortunately, this happens for a traffic demand leading to a blocking probability outside the zone of interest for dimensioning.

The capacity gap of AFC is moderate since it varies from $-12 \%$ to $5 \%$. Moreover, recall that AFC has the multiErlang form. These two observations make AFC suitable in the dimensioning process.

REMARK 1. Tables 2 and 3, give the capacity gaps for cell radii $R=3$ and $5 \mathrm{~km}$ respectively. The results are similar to those obtained for a cell radius $R=0.525 \mathrm{~km}$, except for $R=5 \mathrm{~km}$ and bit-rate $384 \mathrm{kbps}$. Note however that this is an extreme case where a network serves exclusively streaming services at this high bit-rate in a rural-type area (since in an urban area the cell radius doesn't exceed $3 \mathrm{~km}$ ).

\section{CONCLUSION}

We build a multi-Erlang type admission condition for the downlink of a CDMA network, called AFC, which can be a basis of an efficient dimensioning method of this network. It permits also to solve other related problems such as estimating the quality of service perceived by the users of an existing network, or calculate its maximal capacity.

$\mathrm{AFC}$ relies on an approximation of the average power 
transmitted by other base stations. This approximation, based on a mean model is validated by simulations. This intermediate result may be of independent interest when one is interested in the average transmitted power in a CDMA network; e.g. when one aims to know the power remaining for elastic bit-rate (i.e. non-real-time) services, or the effect of a CDMA network on other coexisting networks.

We leave for future work extensions of this approach to the uplink, to elastic bit-rate calls, to other cellular networks such as OFDMA, as well as to the case when the network control needs to cope with user mobility. The numerical results presented in this paper rise also several interesting theoretical questions related to the ordering of the observed blocking probabilities.

\section{Acknowledgment}

The authors are grateful to François Baccelli for his encouragements and many helpful comments. Thanks are also to Salah Eddine Elayoubi for helpful discussions.

\section{REFERENCES}

[1] F. Baccelli, B. Błaszczyszyn, and M. Karray. Up and Downlink Admission/Congestion Control and Maximal Load in Large Homogeneous CDMA Networks. MONET, 9(6), Dec. 2004.

[2] F. Baccelli, B. Błaszczyszyn, and M. Karray. Blocking Rates in Large CDMA Networks via Spatial Erlang Formula. In Proc. of IEEE INFOCOM 2005, 2005.

[3] F. Baccelli, B. Błaszczyszyn, and F. Tournois. Downlink admission/congestion control and maximal load in CDMA networks. In Proc. of IEEE INFOCOM, 2003.

[4] A. Baroudy and S. Elayoubi. HSUPA/HSDPA Systems: Capacity and Dimensioning. Future Generation Communication and Networking, 1, 2007.

[5] B. Błaszczyszyn and M. Karray. Performance Evaluation of Scalable Congestion Control Schemes for Elastic Traffic in Cellular Networks with Power Control. In Proc. of IEEE INFOCOM, 2007.

[6] B. Błaszczyszyn and M. Karray. Impact of mean user speed on blocking and cuts of streaming traffic in cellular networks. In Proc. of European Wireless, 2008.

[7] T. Bonald and A. Proutière. Wireless downlink data channels: user performance and cell dimensioning. In Proc. of MOBICOM, 2003.

[8] T. Chahed, E. Altman, and S. E. Elayoubi. Joint uplink and downlink capacity considerations in admission control in multiservice CDMA/HSDPA systems. In Proc. of ValueTools, 2007.

[9] J. Evans and D. Everitt. On the teletraffic capacity of CDMA cellular networks. IEEE Trans. Vehic. Technol., 48, Jan. 1999.

[10] K. Gilhousen, I. Jacobs, R. Padovani, A. Viterbi, L. Weaver, and C. Wheatley. On the capacity of a cellular CDMA system. IEEE Trans. Vehic. Technol., May 1991.

[11] S. Hanly. Congestion measures in DS-CDMA networks. IEEE Trans. on Comm., 47, Mar. 1999.

[12] H. Holma and A. Toskala. WCDMA for UMTS: Radio Access for Third Generation Mobile Communications. John Wiley and Sons, 2 edition, 2002.

[13] M. Karray. Analytic evaluation of wireless cellular networks performance by a spatial Markov process accounting for their geometry, dynamics and control schemes. PhD thesis, Ecole Nationale Supérieure des Télécommunications, 2007.

[14] J. Kaufman. Blocking in a shared resource environment. IEEE Transactions on Communications, 29(10), 1981.

[15] J. Laiho, A. Wacker, and T. Novosad. Radio Network Planning and Optimisation for UMTS. John Wiley and sons, 2002.

[16] Z. Liu and M. E. Zarki. SIR-based call admission control for DS-CDMA cellular systems. Selected Areas in Communications, IEEE Journal on, 12, May 1994.

[17] J. Roberts. A service system with heterogeneous user requirements. In Performance of Data Communications Systems and their Applications (edited by G. Pujolle), 1981.

[18] A. Viterbi. CDMA: Principles of Spread Spectrum Communications. Addison-Wesley, 1995.

[19] A. Viterbi and A. Viterbi. Erlang capacity of a power controlled CDMA system. IEEE J. Select. Areas Commun., 11(6), Aug. 1993.

[20] J. Zander. Distributed co-channel interference control in cellular radio systems. IEEE Trans. Veh. Technol., 41, 1992.

[21] J. Zander. Performance of optimum transmitter power control in cellular radio systems. IEEE Trans. Vehic. Technol., 41(1), Feb. 1992.

\section{APPENDIX}

We present now more detailed description of the network model as well as explicit expressions of the quantities building the admission conditions considered in the main stream of the paper.

\section{A. BASIC NOTATION}

\section{A.1 Base stations and mobiles}

We model a cellular network as a bounded subset $\mathbb{D}$ of $\mathbb{R}^{2}$ partitioned into cells. $\mathbf{U}$ is the set of base stations (which is assumed finite, but some results may be extended to the infinite case [3]); $u \in \mathbf{U}$ designates a base station. We denote $m \in u$ to designate a user $m$ served by the base station $u$. Depending on the context $u$ and $m$ may denote also the geographical location of the corresponding objects (base stations, mobiles). The propagation-loss between base station $u$ and user $m$ is denoted by $L_{u, m}$.

\section{A.2 Engineering parameters}

$W$ designates the system bandwidth, $N_{0}$ is the power spectral density of external noise. Let $N=W N_{0}$. The bit-rate of user $m$ is denoted by $r_{m}$ and $\xi_{m}$ is the SINR threshold of user $m$. The powers (averaged over fading) are denoted by: $P_{u, m}$ (power transmitted to user $m \in u$ ), $\tilde{P}_{u}$ (maximal total power of each base station), $P_{u}^{\prime}$ (power of common channel, not dedicated to a specific user, which is a fraction of the maximal power $P_{u}^{\prime}=\epsilon \tilde{P}_{u}, \quad u \in \mathbf{U}$ where $\epsilon$ is a given constant), $P_{u}=P_{u}^{\prime}+\sum_{m \in u} P_{u, m}$ (the total power transmitted by base station $u$ ).

The orthogonality factor which affects the intra-cell interference is denoted by $\alpha$. In order to simplify the formulae we introduce

$$
\alpha_{u v}= \begin{cases}1 & \text { if } v \neq u \\ \alpha & \text { if } v=u\end{cases}
$$




\section{A.3 Other useful notation}

In order to simplify the formulae we introduce the $f$-factor

$$
f(m)=\sum_{v \neq u} \frac{L_{u, m}}{L_{v, m}} \frac{\tilde{P}_{v}}{\tilde{P}_{u}}, \quad m \in u
$$

and the modified f-factor

$$
f^{\prime}(m)=\frac{1}{1-\epsilon}\left(\frac{N L_{u, m}}{\tilde{P}_{u}}+\alpha+f(m)\right), \quad m \in u
$$

The modified SINR $\xi_{m}^{\prime}$ is defined by $\xi_{m}^{\prime}=\xi_{m} /\left(1+\alpha \xi_{m}\right)$.

\section{B. NETWORK MODEL}

\section{B.1 Cell pattern}

We consider a simplified model where base stations equipped with omnidirectional antennas are placed on a regular hexagonal grid. (In $\S$ C. 4 we show how the model may be extended to directional antennas.) The distance between two adjacent base stations is denoted by $\Delta$. The cell associated to a given base station is the set of positions which are closest to this base station than any other one. This is in fact a hexagon. We approximate the hexagon by a disc with the same area. The radius of such disc, say $R$, is related to $\Delta$ by $R=\Delta \sqrt{\sqrt{3} /(2 \pi)}$. We call $R$ the cell radius.

If the network is modelled as a bounded region of $\mathbb{R}^{2}$ then the cells at the border of the region experience less interference than those at the center. In this study we are not interested in these border effects, and we consider the network that is wrapped around (i.e., on a torus). In this case all the base stations (or equivalently cells) play the same role since the network is symmetric. We make simulations for hexagonal network on a torus comprising $6 \times 6$ cells. For the Kaufman-Roberts algorithm we further divide each cell into 10 co-centric rings of equal width (equal to $R / 10$ ).

\section{B.2 Propagation-loss}

We assume that the propagation-loss between base station $u$ and user $m$ is given by $L_{u, m}=L(r)=(K r)^{\eta}$ where $K>0$ and $\eta>2$ are given constants and $r$ is the distance between $u$ and $m$. We will not take into account the shadowing effect in the present work. The fading effect is taken into account in the SINR thresholds (given in Table B.1 below)

\section{B.3 F-factor approximation}

For a regular hexagonal network with omnidirectional antennas and the above propagation-loss model the f-factor may be well approximated by the following formula (cf. [13 p.217])

$f(r) \approx \zeta(\eta-1)\left[\frac{L(r)}{L(\Delta-r)}+\frac{L(r)}{L(\Delta+r)}+\frac{4 L(r)}{L\left(\sqrt{\Delta^{2}+r^{2}}\right)}\right]$

where $r$ is the distance between the user and its serving base station, $\zeta$ is the Riemann zeta function given by $\zeta(x)=$ $\sum_{k=1}^{\infty} k^{-x}$.

The above approximation is valid for an infinite network, but may also be used as long as the cell is surrounded by a sufficient number of neighboring cells (typically three rings of cells)

\section{B.4 Traffic classes}

All the formulae in the present paper are valid for the case where there are different traffic classes (each having a specific bit-rate and hence a specific SINR). However the
Table B.1: SINR thresholds in $\mathrm{dB}$ for UMTS vehicular-A channel.

\begin{tabular}{|l||c|c|c|c|}
\hline service & voice & \multicolumn{3}{|c|}{ data } \\
\hline & $12.2 \mathrm{kbps}$ & $64 \mathrm{kbps}$ & $144 \mathrm{kbps}$ & $384 \mathrm{kbps}$ \\
\hline \hline$S I N R(d B)$ & -16 & -11 & -9 & -5 \\
\hline
\end{tabular}

numerical results presented in the present paper concern the case where there is a single traffic class at the same time in the network.

\section{B.5 Fixed numerical parameters}

We consider a UMTS network serving streaming services in the Downlink with omnidirectional antennas. We take a path-loss exponent $\eta=3.38$; a path-loss constant $K=8667$; thermal noise power at mobile $N=-103 \mathrm{dBm}$; base station maximal power (with antenna gain) $\tilde{P}=52 \mathrm{dBm}$; fraction of the maximal power used for common channels $\epsilon=0.12$; orthogonality factor $\alpha=0.4$; bandwidth $W=5 \mathrm{MHz}$. We consider different streaming calls whose SINR thresholds are given in Table B.1 (from [15]) for vehicular-A channel. Note that these values take into account the fading effect (this is why we specify the channel type).

We make simulations for different cell radii $R=0.525,3$ and $5 \mathrm{~km}$. The radius $R=0.525 \mathrm{~km}$ corresponds to a distance between adjacent base stations of $1 \mathrm{~km}$.

\section{EXPLICIT EXPRESSIONS}

\section{C.1 Power allocation problem entries}

It is shown in [13, p.20-22] that the power allocation problem for a CDMA cellular network has the form (2) with

$$
\begin{aligned}
A_{u v} & =\alpha_{u v} \sum_{m \in u} L_{u, m} / L_{v, m} \xi_{m}^{\prime} \\
a_{u} & =\epsilon \tilde{P}_{u}+N \sum_{m \in u} L_{u, m} \xi_{m}^{\prime}
\end{aligned}
$$

where the notation is explained in $\S \mathrm{A}$.

\section{C.2 Mean model}

The parameter $\bar{P}_{0}$ defined in Equation (5) may be viewed as the power in the mean network model introduced in [3] and $[13, \S 4.1]$. This model consists of replacing the matrix $A$ and the vector $a$ intervening in the power allocation problem (2) by their means $E_{\Pi}[A]$ and $E_{\Pi}[a]$ respectively.

For the symmetric model with (spatially) uniform traffic demand it is shown in [13, Eq.(4.2) p.36] that

$$
\bar{P}_{0}=\frac{\bar{M} \bar{\xi}^{\prime} N \bar{L}+\epsilon \tilde{P}}{1-\bar{M} \bar{\xi}^{\prime}(\alpha+\bar{f})}
$$

where for a random variable $Z$ we denote $\bar{Z}$ its expectation, i.e. $\bar{Z}=E[Z]$. More specifically, $\bar{M}$ is the traffic demand per cell in Erlang (added over the different traffic classes); it is related to the traffic demand per surface unit $\rho$ by the relation $\bar{M}=\rho \pi R^{2} . \bar{\xi}^{\prime}$ is the modified SINR averaged over the different traffic classes, where the proportion of each traffic class is defined by the ratio of its traffic demand to the total traffic demand $\bar{M}$ (in this paper only one class of users is considered at a time, thus $\left.\bar{\xi}^{\prime}=\xi^{\prime}\right) . \bar{L}$ is the propagation-loss uniformly averaged over user location in a cell and $\bar{f}$ is the f-factor averaged in the same way.

For the hexagonal model, we have (by an easy direct calculation) $\bar{L}=(1+\eta / 2)^{-1} L(R)$. It is shown also in $[13$, Eq.229] that $\bar{f}$ may be approximated by $\bar{f} \approx 0.9365 /(\eta-2)$. 


\section{C.3 Explicit admission conditions}

The admission conditions SFC, CLC and AFC may be written in the form

$$
\sum_{m \in u} \xi_{m}^{\prime}\left(\alpha+\frac{N L_{u, m}}{\tilde{P}}+\ell \times f(m)\right) \leq 1-\epsilon
$$

where SFC corresponds to $\ell=1$, CLC corresponds to a given constant $\ell \in[0,1]$ (typically $\ell=0.5$ or 0.75 ), AFC corresponds to $\ell=\frac{\bar{P}}{\tilde{P}}$ with $\bar{P}$ is given by (6), or more explicitly, by using (C.2)

$$
\bar{P}=\max \left(\bar{P}_{0}, \tilde{P}\right)=\max \left(\frac{\bar{M} \bar{\xi}^{\prime} N \bar{L}+\epsilon \tilde{P}}{1-\bar{M} \bar{\xi}^{\prime}(\alpha+\bar{f})}, \tilde{P}\right)
$$

\section{C.4 Extension to directional antennas}

Consider a network with directional (three-sector) antennas. The above formulae valid for omnidirectional antennas may be extended to this case with some modifications. We shall use the superscript ${ }^{(D)}$ to designate the parameters for the directional case.

The three sectors situated at a given geographic location are indexed by $1,2,3$. Let $G(\theta)$ be the antenna radiation pattern.

In Equation (C.3) the sum $\sum_{m \in u}$ is now over a cell $u$ which corresponds to a given sector (i.e. to the third of a disc), say sector 1 . The maximal power $\tilde{P}$ accounts now for the directional antenna gain which is generally larger than that of omnidirectional antenna. The path loss accounts now for the antenna radiation pattern, thus $L_{u, m}$ should be replaced by

$$
L_{u, m}^{(D)}=\frac{L(r)}{G(\theta)}
$$

where $(r, \theta)$ are the polar coordinates of $m$ (taking $u$ as origin and the antenna azimuth as angle reference). The f-factor $f(m)$ should be replaced by $f^{(D)}(m)$ given in [13, p.222] and recalled below

$$
f^{(D)}(m)=\frac{\sum_{j=2}^{3} G(\theta-j 2 \pi / D)}{G(\theta)}+\frac{\bar{G}}{G(\theta)} f(r)
$$

where $\bar{G}=\frac{3}{2 \pi} \int_{-\pi}^{\pi} G(\theta) d \theta$; the sum $\sum_{j=2}^{3}$ is over the sectors other than the sector where the user is located; $f(r)$ is the f-factor in the omnidirectional case which may be approximated by (B.1).

In Equation (C.2) $\bar{M}$ is the traffic demand per sector. Moreover $\bar{L}$ and $\bar{f}$ should be replaced respectively by

$$
\begin{aligned}
\bar{L}^{(D)} & =G_{2} \bar{L} \\
\bar{f}^{(D)} & =\bar{G} G_{2} \bar{f}+G_{1}
\end{aligned}
$$

where

$$
\begin{aligned}
G_{1} & =\frac{3}{2 \pi} \int_{-\pi / 3}^{\pi / 3} \frac{\sum_{j=2}^{3} G(\theta-j 2 \pi / D)}{G(\theta)} d \theta \\
G_{2} & =\frac{3}{2 \pi} \int_{-\pi / 3}^{\pi / 3} \frac{1}{G(\theta)} d \theta
\end{aligned}
$$

The numerical values for these constants for typical antenna radiation patterns are given in [13, p.223-224]. 